\title{
"Computersimulation \& Computergraphik" Sommerschule vom 22.-26. September 1997 an der TFH Wildau
}

\author{
Asta Richter
}

Ziel der Sommerschule „Computersimulation \& Computergraphik“ war es, in einem Piloprojekt Kontakte, Wissensaustausch und Vernetzung zwischen verschiedenen Fachdisziplinen über gemeinsam genutzte moderne Medien und Computertechniken zu erreichen. Gleichzeitig wurden praktische Fertigkeiten für die Computersimulation und Computergraphik vermittelt und interessante Anwendungen in Technik und Wirtschaft demonstriert. Es wurde während dieser Woche auf anschauliche und praktische Weise anhand verschiedener Softwarepakete eine Einfuihrung in die komplexe Thematik gegeben und gezeigt, wie Visualisierung auf PCs und im Internet realisiert werden kann.

Die Werbung für die Sommerschule erfolgte durch selbst gestaltete Plakate, Postkarten und das Internet. Anmeldungen konnten sowohl elektronisch über ein Formular im World Wide Web als auch auf konventionellem Wege erfolgen. Die Teilnehmerzahl mußte begrenzt werden, da die Anzahl der Computerarbeitsplätze limitiert war und auch die zur Verfügung stehenden Räume nur eine mittlere Größe hatten. Das Logo der Sommerschule (Abb. 1) - eine Computersimulation von Lyapunovfunktionen mit zwei sich kreuzenden Straßen - veranschaulichte den interdisziplinären Charakter der Veranstaltung.

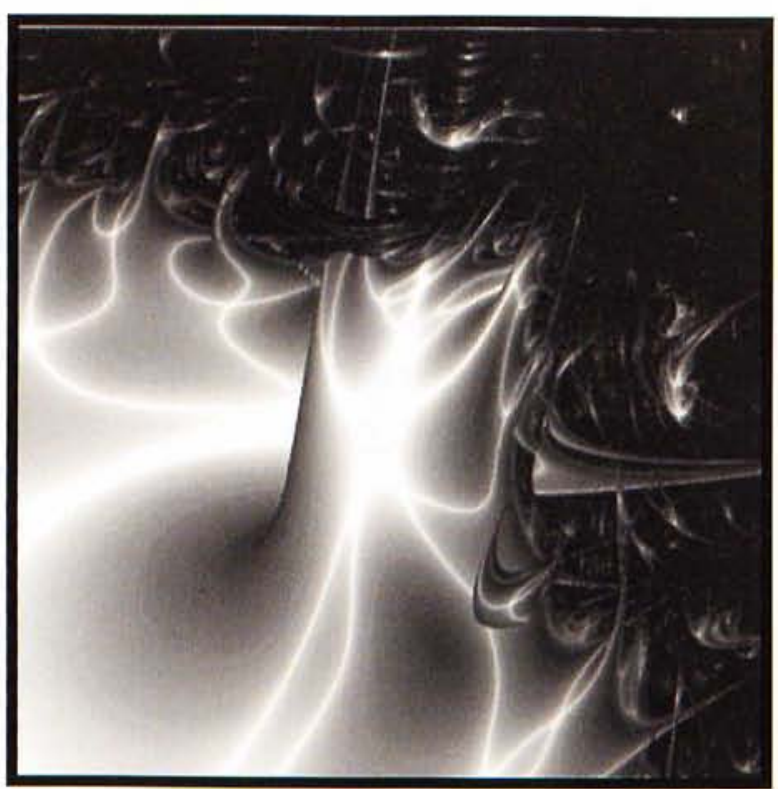

Abb. 1: Logo der Sommerschule

Von den 63 Teilnehmern (Abb. 2 und Tabelle) war etwa die Hälfte Studenten, die andere Hälfte setzte sich aus Mitarbeitern von Firmen, Instituten und Hochschulen zusammen. Mit knapp $10 \%$ hatten wir einen relativ ho-

\begin{tabular}{|l|c|c|c|c|}
\hline \multicolumn{5}{|c|}{$\begin{array}{c}\text { Teilnehmerspektrum der Sommerschule } \\
\text { „Computersimulation \& Computergraphik" }\end{array}$} \\
\hline Teilnehmer & $\begin{array}{c}\text { der TIH } \\
\text { Wildau }\end{array}$ & $\begin{array}{c}\text { aus der } \\
\text { Region }\end{array}$ & $\begin{array}{c}\text { aus dem } \\
\text { Ausland }\end{array}$ & insgesamt \\
\hline gesamt & 34 & 23 & 6 & 63 \\
\hline $\begin{array}{l}\text { Studierende } \\
\text { Mitarbeiter } \\
\text { von Firmen/ } \\
\text { Institutionen }\end{array}$ & 15 & 10 & 5 & 30 \\
\hline Dozenten & 11 & 5 & 1 & 33 \\
\hline Teilnehmerinnen & 6 & 3 & 0 & 9 \\
\hline
\end{tabular}

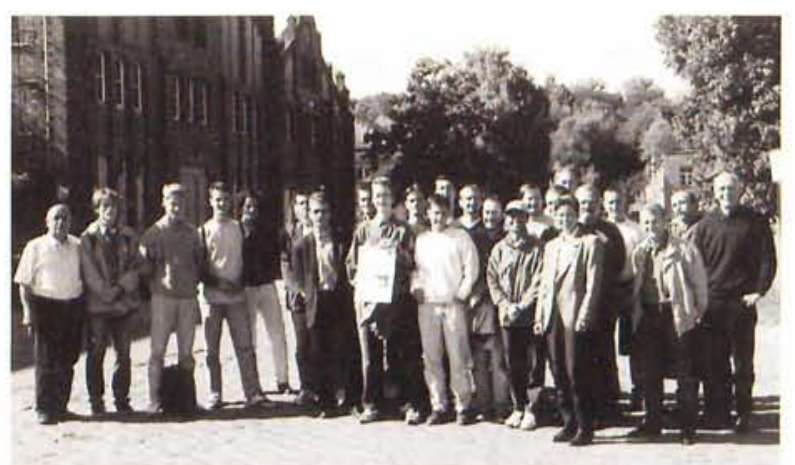

Abb. 2: Foto der Teilnehmer der Sommerschule

hen Anteil von Ausländern an einer lokalen (in deutscher Sprache gehaltenen) Sommerschule. Die Dozenten kamen etwa zu $60 \%$ von der TFH Wildau und zu $40 \%$ von außerhalb. Dies verdeutlicht die Leistungsfähigkeit und Kooperationsbereitschaft der TFH Wildau. Es gelang sogar, Herrn Professor Dr. Roger Smith von der Loughborough University aus England als Dozenten zu gewinnen. Damit konnte ein fachlicher und kultureller Austausch über die Landesgrenzen erreicht werden, der im Rahmen des Sokrates-Programms unterstuitzt wurde. Die Teilnehmer der Sommerschule kamen aus betriebswirtschaftlichen und technischen Fachrichtungen, waren Studierende oder Firmenangehörige, Wissenschaftler an Instituten oder Dozenten von Hochschulen. Damit war die Basis für interessante Diskussionen aus unterschiedlichen Positionen gegeben. Gering war der Anteil der Frauen an der Veranstaltung.

Das Programm der Sommerschule sollte an den Vormittagen einen ausführlichen Einblick in wichtige Programmpakete mit praktischen Übungen am Computer vermitteln. Computerspezialisten aus unterschiedlichen Fachgebieten wie aus der Informatik, Physik, Design, 
Mathematik und dem Ingenieurwesen bildeten ein buntes Dozententeam, welches die jeweilige Thematik aus verschiedenen Blickwinkeln darstellte, was zu völlig neuen und unerwarteten Betrachtungsweisen und Erkenntnissen führte. Simulation und Modellierung künstlicher Realität im Web wurde in der Vorlesung „VRML für Internetanwendungen“ von Herrn Professor Dr. Christian Müller (TFH Wildau) - einem Informatiker - vorgestellt. Aus der Sicht des Physikers wurde PC-Visualisierung und PC-Animation mit dem kostenfreien Programm POV-Ray von unserem englischen Kollegen Professor Dr. Roger Smith (Loughborough University) vorgefuihrt. Ästhetische Vorstellungen spielten eine große Rolle bei der Einführung in das Programmpaket 3D Studio Max zur Modellierung und Visualisierung von 3D-Design durch Herrn Professor Michael Kobzan, einem Designer von der FH Potsdam. Aufbauend auf den strengen Regeln der Mathematik zeigte Herr Dr. Grabow (TFH Wildau) die Leistungsfähigkeit von Mathematica aus der Sicht des Mathematikers. Herrn Prof. Dr.-Ing. habil. B. Hentschel (TFH Wildau) und seinen Mitarbeitern gelang es, ingenieurtechnische Anwendungen in Form eines Boots- und Transportsimulators, Videokonferenztechnik und virtuelle Welten in der Logistik zu präsentieren. Damit wurden vier wichtige Programmpakete mit ihren Vor- und Nachteilen sowie leistungsfähige Anwendungen in Technik und Wirtschaft ausführlich und anschaulich vorgestellt.

Das Fachprogramm der Nachmittage hatte WorkshopCharakter und überstrich einen weiten Bereich der Anwendungen angefangen mit der Modellierung von Molekülen, Baugruppen und mathematischen Strukturen über Prozeßsimulation in der Logistik, Umwelt und in der Wirtschaft bis hin zu Computerkunst und philosophischen Ausfuihrungen und Diskussionen zu Auswirkungen dieser modernen Medien auf unser Leben:

- Prof. Dr. Matthias Forster (TFH Wildau) - „Grundlagen der Verkehrssimulation"

- Prof. Dr. Roger Smith (Loughborough University) und Prof. Dr. Asta Richter (TFH Wildau) - „Simulation von Strukturen aus der physikalischen Welt ${ }^{a}$

- Alexander Häusler (Max-Born-Institut Berlin) - „3DModellierung eines Rastertunnelmikroskops SNOM“

- Prof. Dr. Siegfried Rolle und Dipl.-Ing. Michael Jergović (TFH Wildau) - „Umweltprozeßmodellierung“

- Prof. Michael Kobzan (FH Potsdam) - „Elemente zur Animation in der Werbung “

- Dr. Gerhard Mewes (TFH Wildau) - „Internationale Finanzmärkte im Internet"

- Prof. Dr. László Ungvári und Dipl.-Ing. Astrid Guhl „Kapazitätsplanung im Unternehmen mit Hilfe der Computersimulation"

- Prof. Dr. Udo Hellwig (TFH Wildau) - „Simulation von Feuerungsanlagen"

- Dr. H. Kamusewitz (GKSS Teltow) - „Computergraphik und -simulation in der Membranforschung"

- Dr. Harendt (Institut für Laser- und Plasmatechnik der TFH Wildau) - „Simulation von Gasentladungen“

- Dr. K. Steinmüiller (Institut für Zukunftsforschung Gelsenkirchen) - „Virtuelle Realität - Weg in eine neue Wirklichkeit?"
- Horst Bartnig (freischaffender Künstler Berlin) - Ausstellung und Kïnstlergespräch „Computersimulation für konkrete Kunst".

Beispiele aus dem breiten Spektrum sind in den Abbildungen $3-6$ zu sehen.

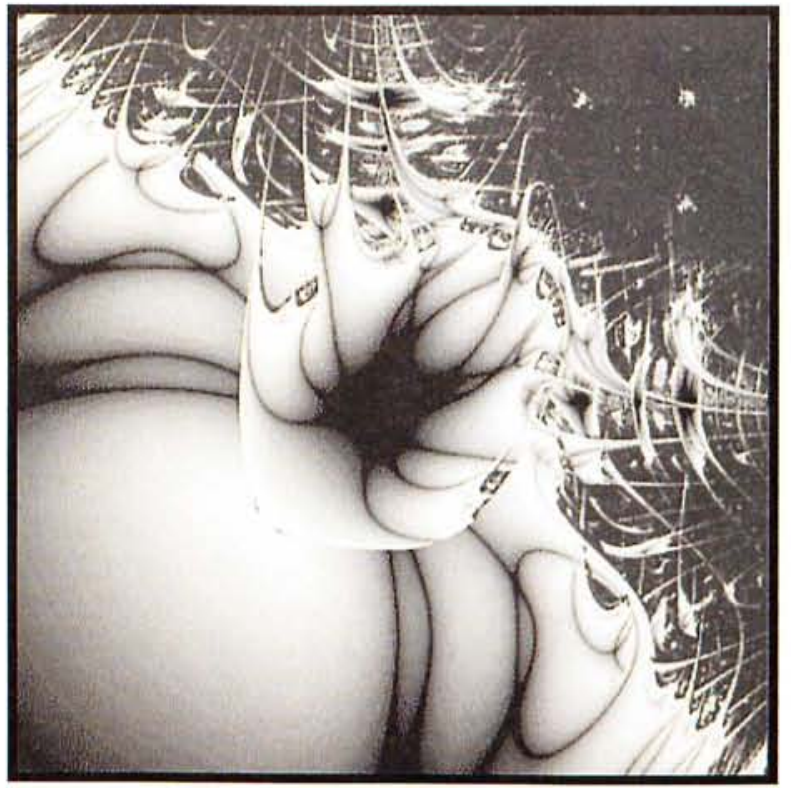

Abb. 3: Lyapunov-Figur „Viscera”
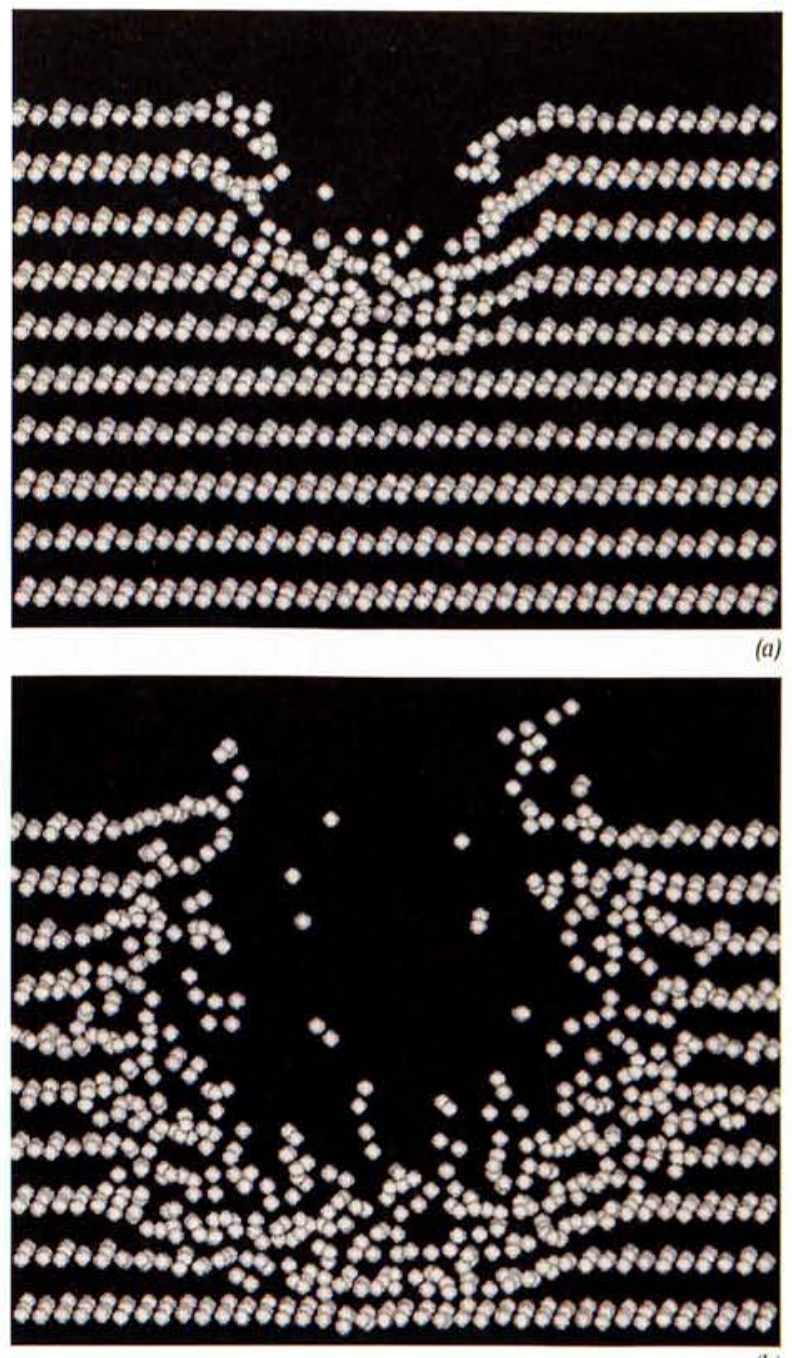

Abb. 4: Simulation von $C_{60}$-lonen-Streuung bei Energien von (b) $300 \mathrm{keV} \mathrm{(a)} \mathrm{und} 600 \mathrm{keV}(\mathrm{b})$. 

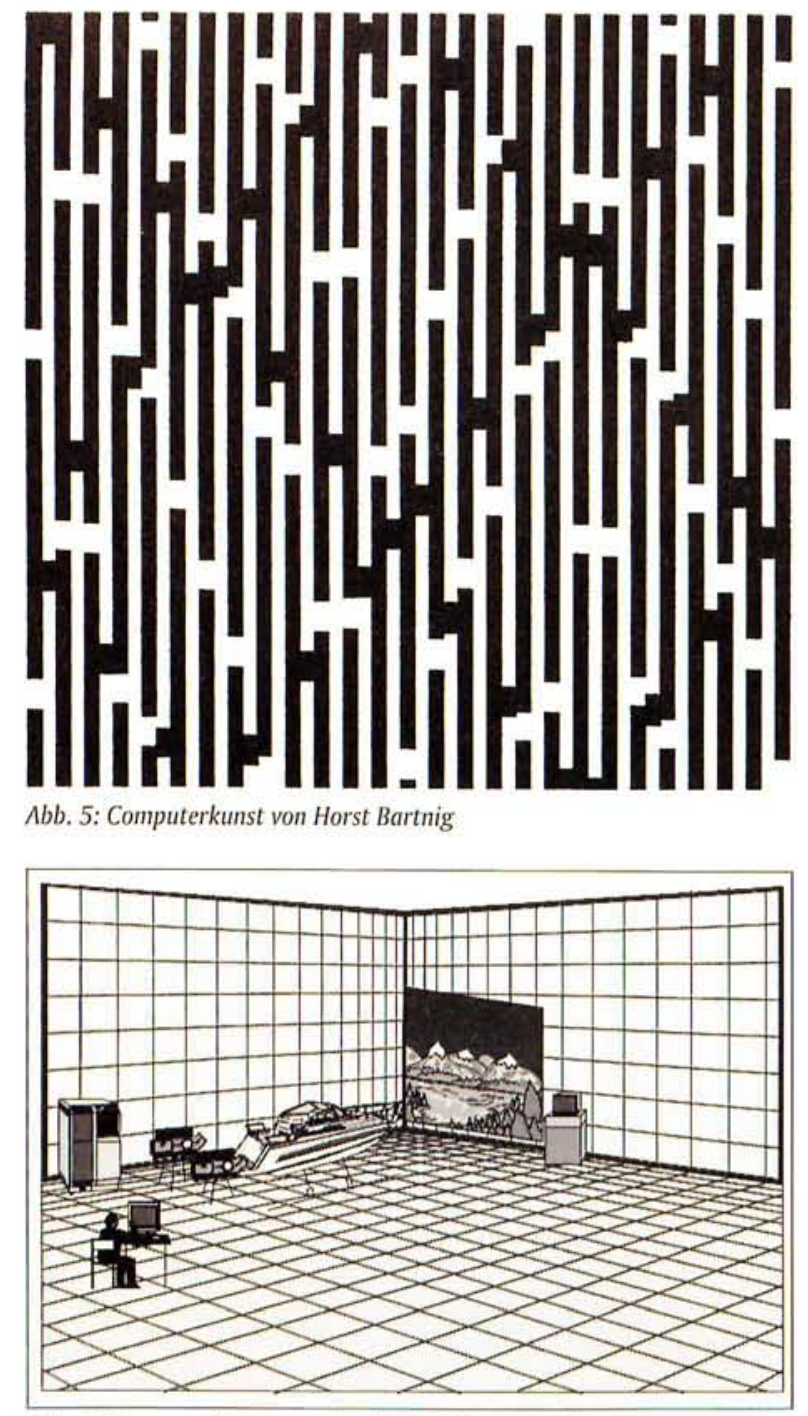

Abb. 6: Boots- und Transportsimulator (Tobias Maechler)

Für die Teilnehmer der Sommerschule wurde ein Skript zu den einzelnen Thematiken erarbeitet, der zum Teil so ausführlich ist, daß ein Selbststudium im Anschluß an die Veranstaltungen mit den entsprechenden Programmpaketen erfolgen kann. An die Teilnehmer der Sommerschule wurden Zertifikate vergeben.

Insbesondere die Ausstellung zur Computerkunst und das Künstlergespräch mit Herrn Horst Bartnig (Abb. 5) als auch die Abschlußdiskussion mit Dr. Karlheinz Steinmüller sprengten den Rahmen des reinen Wissenserwerbs und wiesen auf die gesellschaftlichen Veränderungen und die Verantwortung jedes einzelnen bei der Gestaltung des Informationszeitalters hin.

Besonderer Dank gilt allen Dozenten, die durch thre Beiträge effektiv und anschaulich Fachwissen vermittelten und die Grundlage für interessante Diskussionen legten.

Die technische Ausstattung der Räume für die Durchführung der Sommerschule war sehr gut. Die TFH Wildau stellte leistungsfähige PCs und Projektionssysteme für Computerdisplays (Beamer mit Laptopaufsatz) sowie Videogeräte zur Verfuigung. In mehreren Veranstaltungen wurden die Internet-Verbindungen direkt und inter- aktiv zur Demonstration von den Dozenten und in den Übungen und Anwendung durch die Teilnehmer genutzt. Die einzelnen Veranstaltungen wurden durch das Rechenzentrum der TFH Wildau (Herrn Dipl.-Ing. Miersch und Frau Mattig) und insbesondere die Labormitarbeiter, Herrn Dipl.-Ing. Grau und Herrn Dipl.-Ing. Mieritz, kompetent vorbereitet und mit Engagement technisch betreut. In den Pausen hielt Frau Huget einen kleinen Imbiß und Erfrischungen bereit, der für Stärkung bei dem umfangreichen Programm sorgte und neue Kraft für Diskussionen spendete. Vielen Dank allen Mitarbeitern, die zum Gelingen der Sommerschule beigetragen haben.

Besonderer Dank gilt dem Präsidenten der TFH Wildau, Herrn Prof. Dr. W. Arlt, der die Sommerschule mit einem Grußwort eröffnete, und der Hochschulleitung bei der Unterstützung der Organisation und Durchfuihrung der Sommerschule. Das Ministerium für Wissenschaft, Forschung und Kultur des Landes Brandenburg förderte das Pilotprojekt durch finanzielle Mittel.

\section{Verfasserin}

\section{Prof. Dr. Asta Richter}

Technische Fachhochschule Wildau

Fachbereich Ingenieurwesen/Wirtschaftsingenieurwesen Tel. (0 33 75) 507-219, -217, -184

E-mail: richter@pt.tfh-wildau.de 